\title{
Intraocular pressure I year after vitrectomy in eyes without a history of glaucoma or ocular hypertension
}

This article was published in the following Dove Press journal:

Clinical Ophthalmology

Number of times this article has been viewed

\author{
Yuan Fang' \\ Qingqing Long' \\ Xiaoqian Wang ${ }^{2}$ \\ Rui Jiang' \\ Xinghuai Sun ${ }^{1,3-5}$ \\ 'Department of Ophthalmology and \\ Visual Science, Eye and ENT Hospital, \\ Shanghai Medical College, Fudan \\ University, Shanghai, ${ }^{2}$ Department of \\ Ophthalmology, Jiangyin Bright Eye \\ Hospital, Jiangyin, Jiangsu, ${ }^{3}$ State Key \\ Laboratory of Medical Neurobiology, \\ Institutes of Brain Science and \\ Collaborative Innovation Center for \\ Brain Science, ${ }^{4}$ Key Laboratory of \\ Myopia, NHFPC (Fudan University), \\ ${ }^{5}$ Shanghai Key Laboratory of Visual \\ Impairment and Restoration, Fudan \\ University, Shanghai, People's \\ Republic of China
}

Objective: The aim of this study was to investigate the incidence, risk factors, and treatment of elevated intraocular pressure (IOP) 1 year after vitrectomy in eyes without a history of glaucoma or ocular hypertension.

Patients and methods: This retrospective study comprised 256 eyes from 256 consecutive patients without a history of glaucoma or ocular hypertension who underwent vitrectomy and were followed up for 1 year. The incidence of elevated IOP at 1 year after vitrectomy was calculated. We compared the characteristics of patients with or without elevated IOP to identify possible risk factors for elevated IOP. The treatments used to control IOP were recorded and analyzed. Results: A total of 50 patients (19.5\%) had elevated IOP after vitrectomy at the 1-year follow-up. Tamponade was a significant risk factor for elevated IOP $(P<0.05)$. The cumulative rates of elevated IOP in eyes with air, balanced salt solution, sulfur hexafluoride, perfluoropropane (C3F8), and silicone oil as the tamponade were $0,10.8 \%, 5.9 \%, 19.8 \%$, and $28.4 \%$, respectively $(P<0.05)$. About $68 \%$ of cases of elevated IOP occurred within 1 month after vitrectomy. At 1 year after vitrectomy, 29 patients (58.0\%) had stopped their IOP-lowering drugs and $21(42.0 \%)$ patients were continuing these drugs. About $65 \%$ of ocular hypertension patients who received silicone oil tamponade had not stopped IOP-lowering drugs; this rate was significantly greater than that of ocular hypertension patients who received C3F8 tamponade $(18.2 \%, P<0.05)$.

Conclusion: Elevated IOP is a common complication after vitrectomy. Silicone oil tamponade was associated with greater risk of elevated IOP and had long-term effects on IOP. Drugs and surgery were used to control IOP, and some patients required long-term IOP-lowering therapy. Keywords: ocular hypertension, vitrectomy, silicone oil, glaucoma

\section{Introduction}

Pars plana vitrectomy (PPV) is the most frequently used surgical procedure to treat a variety of retinal disorders. Ocular hypertension is a common complication after PPV. ${ }^{1-7}$ Risk factors for ocular hypertension after PPV include a history of glaucoma, ${ }^{8,9}$ history of diabetes mellitus, ${ }^{8}$ scleral buckling procedures, ${ }^{10-12}$ lensectomy, ${ }^{13-15}$ the use of silicone oil ${ }^{12,16,17}$ or expanding gas, ${ }^{12,17}$ and others. ${ }^{6,18}$ The etiology of ocular hypertension following PPV is complicated, and open-angle and closed-angle mechanisms may be causative factors.

Several studies have investigated that the incidence of ocular hypertension in vitrectomized eyes (including all tamponade methods) varies from $18 \%$ to $28 \%,{ }^{1-4,6}$ and the incidence of elevated intraocular pressure (IOP) after vitrectomy with silicone oil ranges from $20 \%$ to $56 \% .^{5,7}$ Owing to the development of newer equipment and techniques for PPV and lensectomy, as well as changes to the indications for PPV,
Department of Ophthalmology and Visual Science, Eye and ENT Hospital, Shanghai Medical College, Fudan University, 83 Fenyang Road, Shanghai 20003।, People's Republic of China

Tel +862164377134

Fax +86 2164377151

Email xhsun@shmu.edu.cn 
it is important to examine the incidence of ocular hypertension after PPV and identify possible risk factors. In addition, little is known about the long-term prognosis of elevated IOP following PPV. Therefore, the objectives of this study were to analyze the incidence of and risk factors for ocular hypertension after PPV in eyes without a previous history of glaucoma or ocular hypertension and to assess the efficacy of pharmacological and surgical management of ocular hypertension following PPV.

\section{Patients and methods}

This retrospective study comprised 272 consecutive eyes from 272 patients who underwent PPV surgery at the Department of Ophthalmology and Visual Science, Eye and ENT Hospital of Fudan University, in November 2011 and were followed up for 1 year. This study was approved by the institutional review board/ethics committee of the Eye and ENT Hospital of Fudan University and adhered to the ethical standards of the Declaration of Helsinki. This study was explained to each patient, and each patient provided written informed consent for this study.

PPV was performed using a standard $20 \mathrm{G}$ three-port system in all patients. Filtered air, balanced salt solution (BSS), 20\% sulfur hexafluoride (SF6), 14\% perfluoropropane (C3F8), or silicone oil were used as tamponades during vitrectomy. PPV was performed together with lensectomy and scleral bucking in some patients. The patients' demographic, preoperative, intraoperative, and postoperative data were recorded. The preoperative and postoperative data included the best-corrected visual acuity, cup-to-disk ratio, IOP, fundus examination, and ocular medications. The data were recorded at the preoperative visit and postoperatively after PPV at 1 day, 1 week, 1 month after PPV, and monthly thereafter for 1 year. Intraoperative data included the indications for PPV, the tamponade fill during PPV, and the combination of lens surgery or scleral buckling. Ocular hypertension was defined as IOP $\geq 30 \mathrm{mmHg}$ within $24 \mathrm{~h}$ after surgery, ${ }^{12,19}$ or IOP $\geq 25 \mathrm{mmHg}$ any time between postoperative day 2 and 6 weeks after surgery, or IOP $\geq 22 \mathrm{mmHg}$ more than 6 weeks after surgery. ${ }^{20}$ Pharmacotherapy was the primary mode of managing ocular hypertension. Surgery was considered if IOP could not be controlled with maximum tolerable pharmacotherapy. Surgical interventions included Ahmed glaucoma valve implantation, silicone oil removal, and lensectomy.

Data analysis was conducted using SPSS Software (version 18.0; SPSS Inc., Chicago, IL, USA). Descriptive statistics including mean and SD were calculated for patient characteristics. Pearson's $\chi^{2}$ test was used to compare categorical variables, and Student's $t$-test was used to compare continuous variables. Kaplan-Meier survival analysis was used to determine the cumulative rates of ocular hypertension in each tamponade group, and the rates were compared using the Mantel-Cox log-rank test. $P$-values of $<0.05$ were regarded as statistically significant.

\section{Results}

A total of 272 eyes from 272 consecutive patients who underwent PPV were followed up for 1 year. Of these, $16(5.9 \%)$ patients who had a history of glaucoma or ocular hypertension were excluded from the analyses. Therefore, 256 eyes from 256 patients were included in the analyses. A total of 50 patients $(19.5 \%)$ were diagnosed with ocular hypertension on at least one visit. Patients were divided into two groups based on the presence or absence of ocular hypertension.

The demographic characteristics and preoperative data of both groups are summarized in Table 1. Of 50 patients with ocular hypertension, 31 were male (62.0\%) and 19 were female $(38.0 \%)$. Diabetes mellitus was present in 10 patients $(20.0 \%)$, and systemic hypertension was present in six patients $(12.0 \%)$. There were 206 patients without ocular hypertension, of whom 125 were male (60.7\%) and 81 were female $(39.3 \%)$. Diabetes mellitus was present in 39 patients (18.9\%), and systemic hypertension was present in 43 patients $(20.9 \%)$. The mean age was $46.3 \pm 13.3$ years and $50.8 \pm 16.6$ years in patients with elevated IOP and normal IOP, respectively. The refractive error was $-3.6 \pm 5.0 \mathrm{D}$ and $-3.0 \pm 5.6 \mathrm{D}$ in patients with elevated IOP and normal

Table I Demographics of the study population

\begin{tabular}{|c|c|c|c|c|c|}
\hline & \multicolumn{2}{|c|}{$\begin{array}{l}\text { Patients without } \\
\text { elevated IOP }\end{array}$} & \multicolumn{2}{|c|}{$\begin{array}{l}\text { Patients with } \\
\text { elevated IOP }\end{array}$} & \multirow[t]{2}{*}{$P$-value } \\
\hline & $\mathbf{N}=\mathbf{2 0 6}$ & $\%$ & $\mathbf{N}=\mathbf{5 0}$ & $\%$ & \\
\hline \multicolumn{5}{|l|}{ Age (years) } & $0.192 *$ \\
\hline Mean \pm SD & \multicolumn{2}{|l|}{$50.8 \pm 16.6$} & \multicolumn{2}{|l|}{$46.3 \pm 13.3$} & \\
\hline Range & \multicolumn{2}{|l|}{$5-80$} & \multicolumn{2}{|l|}{$9-67$} & \\
\hline \multicolumn{5}{|c|}{ Preoperative IOP $(\mathrm{mmHg})$} & $0.980 *$ \\
\hline Mean \pm SD & $12.6 \pm 4 . \mid$ & & $12.9 \pm 4.2$ & & \\
\hline Range & $5-20$ & & $3-21$ & & \\
\hline \multicolumn{5}{|l|}{ Diopter (D) } & $0.97 I^{*}$ \\
\hline Mean \pm SD & $-3.0 \pm 5.6$ & & $-3.6 \pm 5.0$ & & \\
\hline Range & -27 to 2 & & -14 to 2 & & \\
\hline \multicolumn{5}{|l|}{ Gender } & $0.864^{\dagger}$ \\
\hline Male & 125 & $60.68 \%$ & 31 & $62.00 \%$ & \\
\hline Female & 81 & $39.32 \%$ & 19 & $38.00 \%$ & \\
\hline \multicolumn{6}{|l|}{ Medical history } \\
\hline DM & 39 & $18.93 \%$ & 10 & $20.00 \%$ & $0.863^{\dagger}$ \\
\hline HP & 43 & $20.87 \%$ & 6 & $12.00 \%$ & $0.153^{\dagger}$ \\
\hline
\end{tabular}

Notes: ${ }^{*} P$-value by independent $t$-test. ${ }^{\dagger} P$-value by Pearson's $\chi^{2}$ test. Abbreviations: DM, diabetes mellitus; HP, hypertension; IOP, intraocular pressure. 
IOP, respectively. The patient demographic characteristics, including age, gender, history of systemic diseases, and refractive degree were not significantly different between the two groups of patients. The mean preoperative IOP was $12.9 \pm 4.2$ and $12.6 \pm 4.1 \mathrm{mmHg}$ in patients with elevated IOP and normal IOP, respectively. The preoperative IOP was not significantly different between the two groups $(P=0.980$, independent $t$-test).

The intraoperative characteristics of patients are summarized in Table 2. In patients with elevated IOP, the underlying ocular diseases necessitating PPV included macular diseases $(2.0 \%)$, rhegmatogenous retinal detachment (48.0\%), proliferative diabetic retinopathy (16.0\%), traumarelated vitreous retinal diseases $(22.0 \%)$, vitreous hemorrhage of nondiabetic etiology $(4.0 \%)$, and other diseases $(8.0 \%)$. The types of ocular disease were not significantly different between the two groups ( $P=0.181$, Pearson's $\chi^{2}$ test $)$. However, the incidence of elevated IOP was 3.7\% (one of 27 patients) in patients with macular diseases versus $21.4 \%$ (49 of 229 patients) in patients with non-macular diseases (including other diseases), and this difference was statistically significant $\left(P=0.028\right.$, Pearson's $\chi^{2}$ test). Tamponade was

Table 2 Intraoperative characteristics of patients with and without elevated IOP

\begin{tabular}{|c|c|c|c|c|c|}
\hline & \multicolumn{2}{|c|}{$\begin{array}{l}\text { Patients } \\
\text { without } \\
\text { elevated IOP }\end{array}$} & \multicolumn{2}{|c|}{$\begin{array}{l}\text { Patients } \\
\text { with } \\
\text { elevated IOP }\end{array}$} & \multirow[t]{2}{*}{$P$-value } \\
\hline & $\mathbf{N}=\mathbf{2 0 6}$ & $\%$ & $\mathbf{N}=\mathbf{5 0}$ & $\%$ & \\
\hline \multicolumn{5}{|l|}{ Diagnosis } & $0.18 I^{*}$ \\
\hline Macular diseases & 26 & 12.62 & 1 & 2.00 & \\
\hline Vitreous hemorrhage & 19 & 9.22 & 2 & 4.00 & \\
\hline Retinal detachment & 91 & 44.17 & 24 & 48.00 & \\
\hline Trauma related & 40 & 19.42 & 11 & 22.00 & \\
\hline PDR & 24 & 11.65 & 8 & 16.00 & \\
\hline Others & 6 & 2.91 & 4 & 8.00 & \\
\hline \multicolumn{5}{|l|}{ Tamponade } & $0.036 *$ \\
\hline Air & 10 & 4.85 & 0 & 0.00 & \\
\hline BSS & 33 & 16.02 & 4 & 8.00 & \\
\hline SF6 & 16 & 7.77 & 1 & 2.00 & \\
\hline C3F8 & 89 & 43.20 & 22 & 44.00 & \\
\hline Silicone oil & 58 & 28.16 & 23 & 46.00 & \\
\hline \multicolumn{5}{|l|}{ Combined lens surgery } & $0.336 *$ \\
\hline Without lens surgery & 133 & 64.56 & 35 & 70.00 & \\
\hline Lensectomy & 43 & 20.87 & 12 & 24.00 & \\
\hline Lensectomy + IOL & 27 & 13.11 & 2 & 4.00 & \\
\hline IOL removal & 3 & 1.46 & I & 2.00 & \\
\hline \multicolumn{5}{|l|}{ Combined scleral buckling } & $0.54 I^{*}$ \\
\hline Without SB & 191 & 92.72 & 48 & 96.00 & \\
\hline With SB & 15 & 7.28 & 2 & 4.00 & \\
\hline
\end{tabular}

Note: $* P$-value by Pearson's $\chi^{2}$ test.

Abbreviations: SF6, sulfur hexafluoride; C3F8, perfluoropropane; BSS, balanced salt solution; IOL, intraocular lens; IOP, intraocular pressure; PDR, proliferative diabetic retinitis; SB, scleral buckling. provided by BSS, 20\% SF6, 14\% C3F8, and silicone oil in $8.0 \%, 2.0 \%, 44.0 \%$, and $46.0 \%$ of patients with elevated IOP, respectively. Tamponade was provided by filtered air, BSS, $20 \%$ SF6, 14\% C3F8, and silicone oil in 4.9\%, 16.0\%, 7.8\%, $43.2 \%$, and $28.2 \%$ of patients with normal IOP, respectively. The type of tamponade was significantly different between the two groups $\left(P=0.036\right.$, Pearson's $\chi^{2}$ test $)$.

PPV was performed together with lens surgery in some patients. Among 50 patients with elevated IOP, 35 (70.0\%) underwent PPV without lens surgery, 12 (24.0\%) underwent PPV with lensectomy, two (4.0\%) underwent PPV with lensectomy and intraocular lens (IOL) implantation, and one $(2.0 \%)$ underwent PPV with IOL removal. Among 206 patients with normal IOP, 133 (64.6\%) underwent PPV without lens surgery, 43 (20.9\%) underwent PPV with lensectomy, 27 (13.1\%) underwent PPV with lensectomy and IOL implantation, and three (1.5\%) underwent PPV with IOL removal. The distribution of surgical procedures was not significantly different between the two groups $(P=0.336$, Pearson's $\chi^{2}$ test).

PPV was performed in combination with scleral buckling in $2 / 50(4.0 \%)$ patients with elevated IOP compared with $15 / 206(7.3 \%)$ patients with normal IOP. The proportion of patients who underwent PPV together with scleral buckling was not significantly different between the two groups $\left(P=0.541\right.$, Pearson's $\chi^{2}$ test $)$.

After analyzing the risk factors for elevated IOP based on the baseline and surgical procedures as listed in Tables 1 and 2, only PPV tamponade type was a significant risk factor for elevated IOP. We compared the total proportion of eyes with elevated IOP in the different tamponade groups as shown in Figure 1. The number of patients in the air group, the BSS group, and the SF6 group was small; therefore, we combined these three groups with the others group in this comparison. As shown in Figure 1, the total proportion of eyes with elevated IOP in the silicone oil group, the C3F8 group, and the others group was $28.4 \%, 19.8 \%$, and $7.8 \%$, respectively. The difference between the silicone oil group and the others group was significant $(P=0.002$, Pearson's $\chi^{2}$ test). There was also a significant difference between the C3F8 group and the others group $(P=0.034$, Pearson's $\chi^{2}$ test). There was no significant difference between the C3F8 group and the silicone oil group $(P=0.166$, Pearson's $\chi^{2}$ test). To analyze the association between tamponade and elevated IOP over time, the onset and duration of elevated IOP according to the type of tamponade was examined, and the results are presented in Table 3 and Figure 2. As summarized in Table 3, the elevated IOP was detected at 


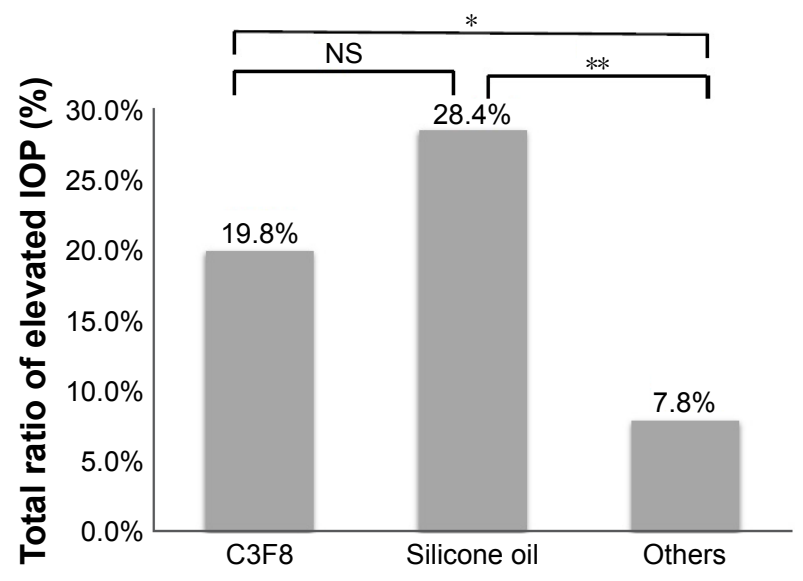

Figure I Comparison of the total proportion of eyes with elevated IOP among the different tamponade groups.

Notes: The proportion of eyes with elevated IOP was significantly higher in the silicone oil group than in the others group (combined air, BSS, and SF6 groups; $P=0.002$, Pearson's $\chi^{2}$ test). The proportion of eyes with elevated IOP was significantly higher in the C3F8 group than in the others group ( $P=0.034$, Pearson's $\chi^{2}$ test). There was no significant difference in the proportion of eyes with elevated IOP between the silicone oil group and the C3F8 group ( $P=0.166$, Pearson's $\chi^{2}$ test). *Significant; **highly significant.

Abbreviations: SF6, sulfur hexafluoride; C3F8, perfluoropropane; BSS, balanced salt solution; IOP, intraocular pressure; NS, not significant.

1 week after surgery in $10(20.0 \%)$ patients, at 1 month in $34(68.0 \%)$ patients, at 3 months in $41(82.0 \%)$ patients, at 4-6 months in two (4.0\%) patients, at 7-9 months in one (2.0\%) patient, and at 10-12 months in six (12.0\%) patients. Therefore, most of the patients experienced elevated IOP within 3 months after PPV. Fewer new cases of elevated IOP occurred at 4-9 months during the first year after PPV, and more new cases occurred at 10-12 months. In patients whose tamponade was provided by BSS or SF6, there were no new cases of elevated IOP beyond 3 months after surgery. By contrast, some patients whose tamponade was provided by $\mathrm{C} 3 \mathrm{~F} 8$ or silicone oil experienced elevated IOP more than 3 months after surgery. We used Kaplan-Meier analysis to compare the cumulative rates of elevated IOP according to the type of tamponade. As shown in Figure 2, the cumulative rates of elevated IOP at 12 months after PPV were 28.4\%,

Table 3 Number of eyes with elevated IOP according to tamponade type and time of onset

\begin{tabular}{llllllll}
\hline & BSS & Air & SF6 & C3F8 & Oil & Total & Cumulative\% \\
\hline $0-1 \mathrm{w}$ & $\mathrm{I}$ & 0 & $\mathrm{I}$ & 4 & 4 & 10 & $20.00 \%$ \\
$\mathrm{I} \mathrm{w}-\mathrm{I} \mathrm{m}$ & 2 & 0 & 0 & 12 & 10 & 24 & $68.00 \%$ \\
$\mathrm{I} \mathrm{m}-3 \mathrm{~m}$ & $\mathrm{I}$ & 0 & 0 & 3 & 3 & 7 & $82.00 \%$ \\
$4 \mathrm{~m}-6 \mathrm{~m}$ & 0 & 0 & 0 & 0 & 2 & 2 & $86.00 \%$ \\
$7 \mathrm{~m}-9 \mathrm{~m}$ & 0 & 0 & 0 & 0 & $\mathrm{I}$ & $\mathrm{I}$ & $88.00 \%$ \\
$10 \mathrm{~m}-12 \mathrm{~m}$ & 0 & 0 & 0 & 3 & 3 & 6 & $100.00 \%$ \\
Total & 4 & 0 & $\mathrm{I}$ & 22 & 23 & 50 & \\
\hline
\end{tabular}

Notes: Air, filtered air; oil, silicone oil.

Abbreviations: SF6, sulfur hexafluoride; C3F8, perfluoropropane; BSS, balanced salt solution; IOP, intraocular pressure; m, month; w, week.

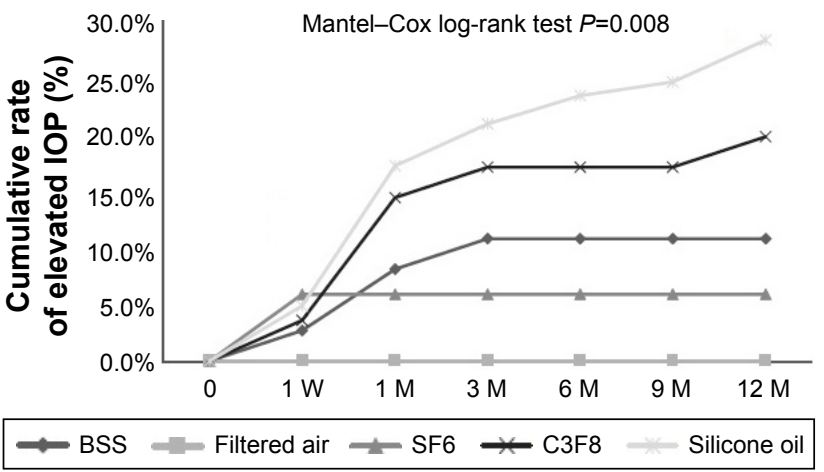

Figure 2 Survival analysis of elevated IOP according to the type of tamponade used in PPV.

Note: The cumulative rate of elevated IOP was significantly different among the five types of tamponade $(P=0.008$, log-rank test) and was greatest for silicone oil tamponade.

Abbreviations: SF6, sulfur hexafluoride; C3F8, perfluoropropane; BSS, balanced salt solution; IOP, intraocular pressure; M, month; PPV, pars plana vitrectomy; W, week.

$19.8 \%, 10.8 \%, 5.9 \%$, and $0 \%$ for silicone oil, C3F8, BSS, SF6, and air, respectively. The cumulative rate of elevated IOP was significantly different between these types of tamponade ( $P=0.008$, Mantel-Cox log-rank test $)$ indicating that the silicone oil tamponade was associated with the greatest risk of elevated IOP, followed by C3F8, SF6, BSS, and air. Therefore, silicone oil and C3F8 tamponades have a greater risk for elevated IOP compared with SF6, BSS, and air tamponades.

Pharmacotherapy was the primary mode of treating elevated IOP. As indicated in Table 4, only topical and systemic IOP-lowering drugs were prescribed in $31 / 50(62.0 \%)$ patients. These drugs were prescribed for temporary use in $21 / 50(42.0 \%)$ patients, and for persistent use in $10 / 50$ $(20.0 \%)$ patients. Surgical interventions to control IOP, including Ahmed glaucoma valve implantation, lensectomy, and silicone oil removal, were used in 15/50 (30.0\%) patients. These procedures were successful (ie, achieved a normal IOP without IOP-lowering drugs) in five eyes (10.0\%) and were associated with qualified success (ie, achieved normal IOP with IOP-lowering drugs) in 10 eyes (20.0\%).

Table 4 Treatment outcomes of IOP elevation according to tamponade type

\begin{tabular}{|c|c|c|c|c|c|}
\hline & \multicolumn{2}{|l|}{ Drugs alone } & \multicolumn{2}{|l|}{ Surgery } & \multirow{2}{*}{$\begin{array}{l}\text { Drop } \\
\text { treatment }\end{array}$} \\
\hline & Temporary & Persistent & $\begin{array}{l}\text { Complete } \\
\text { success }\end{array}$ & $\begin{array}{l}\text { Qualified } \\
\text { success }\end{array}$ & \\
\hline BSS & 2 (50.00\%) & $2(50.00 \%)$ & 0 & 0 & 0 \\
\hline SF6 & I (I00\%) & 0 & 0 & 0 & 0 \\
\hline C3F8 & $15(68.18 \%)$ & $3(13.63 \%)$ & I (4.55\%) & 0 & 3 (13.63\%) \\
\hline Oil & 3 (I3.04\%) & 5 (21.74\%) & 4 (17.39\%) & 10 (43.48\%) & I (4.35\%) \\
\hline Total & 21 (42.00\%) & 10 (20.00\%) & $5(10.00 \%)$ & $10(20.00 \%)$ & 4 (8.00\%) \\
\hline
\end{tabular}

Notes: Results are presented as N (\%). Oil, silicone oil.

Abbreviations: SF6, sulfur hexafluoride; C3F8, perfluoropropane; BSS, balanced salt solution; IOP, intraocular pressure. 
Table 5 Outcomes of surgical procedures for the control of IOP

\begin{tabular}{|c|c|c|c|c|c|c|}
\hline & \multicolumn{2}{|c|}{$\begin{array}{l}\text { Complete } \\
\text { success }\end{array}$} & \multicolumn{2}{|c|}{$\begin{array}{l}\text { Qualified } \\
\text { success }\end{array}$} & \multicolumn{2}{|c|}{ Failure } \\
\hline & $\mathbf{N}$ & $\%$ & $\mathbf{N}$ & $\%$ & $\mathbf{N}$ & $\%$ \\
\hline Valve implantation & 3 & 100 & 0 & 0 & 0 & 0 \\
\hline Silicone oil removal & 1 & 8.33 & 10 & 83.33 & I & 8.33 \\
\hline Lensectomy & 1 & 100 & 0 & 0 & 0 & 0 \\
\hline
\end{tabular}

Abbreviation: IOP, intraocular pressure.

In $4 / 50(8.0 \%)$ eyes with elevated IOP, the IOP-lowering drugs were stopped because of near-total loss of vision; three of these eyes received $\mathrm{C} 3 \mathrm{~F} 8$ tamponade and one received silicone oil tamponade.

The outcomes of treating elevated IOP according to the type of tamponade are presented in Table 4. IOP-lowering drugs were prescribed for temporary use in $100.0 \%, 50.0 \%$, $68.2 \%$, and $13.0 \%$ of patients who received SF6, BSS, C3F8, and silicone oil tamponades, respectively. The proportion of patients on long-term IOP-lowering drug use (including patients with persistent use of IOP-lowering drugs and patients with qualified success) was significantly different between the types of tamponade ( $P=0.004$, Pearson's $\chi^{2}$ test). These results suggest that the duration of IOP-lowering drug use differs between different types of tamponade. Silicone oil typically causes persistent damage to the aqueous outflow pathways and most patients who developed ocular hypertension after silicone oil tamponade required long-term administration of IOP-lowering drugs. By contrast, although $\mathrm{C} 3 \mathrm{~F} 8$ is associated with damage to the aqueous outflow pathways, these pathways recover over time necessitating short-term administration of IOP-lowering drugs.

Three patients $(6.0 \%)$ with elevated IOP underwent Ahmed valve implantation, which kept IOP within the normal range. These three patients received silicone oil tamponade. One patient with C3F8 tamponade underwent lensectomy, which was successful. Among 23 patients with elevated IOP after silicone oil tamponade, 12 underwent surgery to remove silicone oil and reduce IOP within 1 year after PPV. As summarized in Table 5, after silicone oil removal, one patient achieved normal IOP without IOP-lowering drugs, 10 patients achieved normal IOP with IOP-lowering drugs, and one patient could not achieve normal IOP with IOPlowering drugs. This patient underwent Ahmed valve implantation, which resulted in normal IOP.

\section{Discussion}

IOP elevation is one of the most common and severe complications of PPV. Elevated IOP was reported to affect $20 \%-60 \%$ of eyes after PPV. ${ }^{1-5}$ Various definitions of elevated
IOP have been used in previous studies. In our study, ocular hypertension was defined as IOP $\geq 30 \mathrm{mmHg}$ at $24 \mathrm{~h}$ after surgery, ${ }^{12,19}$ IOP $\geq 25 \mathrm{mmHg}$ between postoperative day 2 and 6 weeks after surgery, or IOP $\geq 22 \mathrm{mmHg}$ beyond 6 weeks after surgery and required treatment. ${ }^{20}$

Many risk factors for elevated IOP after PPV were reported in previous studies. In particular, preexisting glaucoma is a very important risk factor for elevated IOP after PPV. ${ }^{8,9}$ Phelps and Burton ${ }^{19}$ reported that among 817 patients, glaucoma preceded retinal detachment historically or based on clinical evidence in nearly $7 \%$ of patients. In our original cohort of 272 patients who underwent PPV, 5.9\% had a history of glaucoma or ocular hypertension, which is similar to the value in their report. In fact, some patients with preexisting glaucoma were misdiagnosed before PPV. Therefore, the actual prevalence of glaucoma among patients undergoing PPV might exceed 5.9\%. To detect risk factors for elevated IOP, other than the history of glaucoma or ocular hypertension, we excluded patients with a history of glaucoma or ocular hypertension from our study.

In our study, the cumulative rate of ocular hypertension after PPV in patients without a history of glaucoma or ocular hypertension was $19.5 \%$, which is similar to the values reported elsewhere. ${ }^{17,21}$ Ocular hypertension was detected within 1 month after PPV in $68.0 \%$ of patients with elevated IOP, consistent with another study. ${ }^{22}$

Postoperative hemorrhage, inflammation, and pupillary block might contribute to early-onset ocular hypertension. Late-onset ocular hypertension may be caused by anterior synechiae, rubeosis iridis, migration of silicone oil into the anterior chamber, and long-term steroid use. ${ }^{23-25}$ In previous studies, the risk factors for ocular hypertension after PPV included the history of glaucoma,${ }^{8}$ history of diabetes mellitus, ${ }^{8,12}$ the use of silicone oil and expanding gas tamponade, ${ }^{12,16,17}$ PPV combined with lensectomy, ${ }^{13-15}$ and scleral buckling. ${ }^{10-12}$ In our study, we excluded patients with a history of glaucoma to detect other risk factors and found that only tamponade was a significant risk factor for elevated IOP after PPV. The cumulative rates of ocular hypertension at 1 year after PPV were 28.4\%, 19.8\%, 10.8\%, $5.9 \%$, and $0 \%$ for silicone oil, C3F8, BSS, SF6, and air, respectively. In patients who received silicone oil tamponade, the incidence of ocular hypertension continued to increase during the 1-year follow-up. By contrast, in patients who received SF6 or BSS tamponade, there were no new cases of ocular hypertension more than 3 months after PPV. The mechanism of IOP elevation varied among the different tamponade types. Preexisting glaucoma, steroid-induced ocular hypertension, and postoperative inflammation were 
common mechanisms in the C3F8 and silicone oil groups. Furthermore, the migration of emulsified silicone oil into the anterior chamber might block or infiltrate the trabecular meshwork to cause angle-closure glaucoma or open-angle glaucoma with trabeculitis. ${ }^{24,26}$

The duration of ocular hypertension also differed among the tamponade types. Of note, most cases of ocular hypertension were persistent in patients who received silicone oil tamponade and most of these patients continued to use IOP-lowering drugs until the last visit at 1 year after surgery, whereas most cases of ocular hypertension were temporary in patients who received $\mathrm{C} 3 \mathrm{~F} 8$ tamponade. These results indicate that the type of tamponade is a significant determinant of the incidence and duration of ocular hypertension after PPV.

There are conflicting reports on whether lensectomy is a risk factor for ocular hypertension. ${ }^{13,14,27-29}$ In one long-term study of patients with late-onset open-angle hypertension or glaucoma after PPV, the incidence of late-onset open-angle hypertension was $15 \%$ in nonphakic eyes versus $1.4 \%$ in phakic eyes $(P<0.05) .{ }^{13}$ In another study, the incidence of late-onset open-angle glaucoma was $2 \%$ in phakic eyes versus $13 \%$ in pseudophakic eyes $(P<0.05) .{ }^{14}$ The results of these studies suggest that the presence of a crystalline lens reduces the risk of open-angle glaucoma, and that lensectomy is a risk factor for late-onset open-angle glaucoma. However, in another study of late-onset open-angle glaucoma or ocular hypertension after simple PPV with or without lens surgery, lens surgery was not a risk factor for late-onset open-angle glaucoma. ${ }^{29}$ Moreover, another study that examined the incidence of elevated IOP at $48 \mathrm{~h}$ after simple PPV combined with or without lensectomy and IOL implantation suggested that lensectomy is a risk factor for postoperative short-term ocular hypertension. ${ }^{15}$ However, in the current study, the incidence of elevated IOP was not significantly different between patients who did or did not undergo lens surgery. We speculate that, although lensectomy in combination with PPV might increase postoperative inflammation and the risk of open-angle ocular hypertension, it could decrease the risk of pupil block, which leads to closed-angle ocular hypertension especially when expanding gas or silicone oil is used as the tamponade in PPV. This might explain why lensectomy was not a significant risk factor for ocular hypertension after PPV in our study.

Once ocular hypertension was detected, the patient was treated pharmacologically or surgically to lower IOP. Surgical procedures to lower IOP in this study included silicone oil removal, Ahmed valve implantation, and lensectomy.
Overall, 14/23 eyes with elevated IOP after silicone oil tamponade underwent IOP-lowering surgery compared with $1 / 22$ eyes that received C3F8 tamponade. About $65 \%$ of eyes with elevated IOP after silicone oil tamponade required persistent administration of IOP-lowering drugs compared with $14 \%$ of eyes after C3F 8 tamponade. These results highlight the different clinical outcomes of various tamponades used in PPV.

In the study by Budenz et al, ${ }^{30}$ silicone oil removal alone was associated with an overall success rate of $62.5 \%$ (including complete success and qualified success) for reducing silicone oil-related ocular hypertension. In a study by Nguyen et al, ${ }^{21}$ silicone oil removal alone had a success rate of $57.1 \%$. In the current study, silicone oil removal surgery was performed in 12 patients to control IOP. This procedure was defined as complete success in one $(8.3 \%)$ patient, as qualified success in $10(83.3 \%)$ patients, and was unsuccessful in one $(8.3 \%)$ patient. This patient underwent Ahmed glaucoma valve implantation, which controlled IOP. Therefore, the overall success rate (including complete success and qualified success) of silicone oil removal to control IOP was higher in our study than in earlier studies. ${ }^{30}$ The higher success rate of silicone oil removal to control IOP in our study could be related to the introduction of newer IOP-lowering drugs, particularly prostaglandin analogs, in recent years.

\section{Conclusion}

In this study, tamponade, especially with expanding gas or silicone oil, was the only significant risk factor for ocular hypertension after PPV in patients without a history of glaucoma. Lensectomy and scleral buckling were not significant risk factors for ocular hypertension after PPV in our study. The cumulative rate of ocular hypertension was higher in patients who received silicone oil tamponade than in patients who received other types of tamponade. Patients with ocular hypertension after PPV with silicone oil tamponade required long-term treatment with IOP-lowering drugs or surgery. Thus, patients who undergo PPV with silicone oil tamponade require long-term monitoring of IOP to detect ocular hypertension. The shortcoming of this study is the lack of angle description of ocular hypertension. Our study should help clinicians to estimate the risk and management of ocular hypertension after PPV. The prospective investigations with longer follow-up may strengthen our results in the future.

\section{Acknowledgments}

This study was supported by the Chinese National Natural Science Foundation (NSFC81100667) and Chinese 
International Science and Technology Cooperation Program (No 2015DFA31340). Dr Yuan Fang and Dr Qingqing Long are co-first authors for this study.

\section{Disclosure}

The authors report no conflicts of interest in this work.

\section{References}

1. Faulbom J, Conway BP, Machemer R. Surgical complications of pars plana vitreous surgery. Ophthalmology. 1978;85(2):116-125.

2. Weinberg RS, Peyman GA, Huamonte FU. Elevation of intraocular pressure after pars plana vitrectomy. Albrecht Von Graefes Arch Klin Exp Ophthalmol. 1976;200(2):157-161.

3. Aaberg TM, VanHorn DL. Late complications of pars plana vitrectomy surgery. Ophthalmology. 1978;85(2):126-140.

4. Ghartey KN, Tolentino FE, Freeman HM, McMeel JW, Schepens CL, Aiello LM. Closed vitreous surgery. XVII. Results and complications of pars plana vitrectomy. Arch Ophthalmol. 1980;98:1248-1252.

5. Antoun J, Azar G, Jabbour E, et al. Vitreoretinal surgery with silicone oil tamponade in primary uncomplicated rhegmatogenous retinal detachment. Retina. 2016;36(10):1906-1912.

6. Parke DW 3rd, Sisk RA, Houston SK, Murray TG. Ocular hypertension after intravitreal triamcinolone with vitrectomy and phacoemulsification. Clin Ophthalmol. 2012;6:925-931.

7. Jonas JB, Knorr HL, Rank RM, Budde WM. Intraocular pressure and silicone oil endotamponade. J Glaucoma. 2001;10(2):102-108.

8. Henderer JD, Budenz DL, Flynn HW, Schiffman JC, Feuer WJ, Murray TG. Elevated intraocular pressure and hypotony following silicone oil retinal tamponade for complex retinal detachment. Arch Ophthalmol. 1999;117(2):189-195.

9. Wu L, Berrocal MH, Rodriguez FJ, et al. Intraocular pressure elevation after uncomplicated pars plana vitrectomy: results of the Pan American Collaborative Retina Study Group. Retina. 2014;34(10):1985-1989.

10. Hartley RE, Marsh RJ. Anterior chamber depth changes after retinal detachment. Br J Ophthalmol. 1973;57(8):546-550.

11. Kreiger AE, Hodgkinson BJ, Frederick AR Jr, Smith TR. The results of retinal detachment surgery. Analysis of 268 operations with a broad scleral buckle. Arch Ophthalmol. 1971;86(4):385-394.

12. Muether PS, Hoerster R, Kirchhof B, Fauser S. Course of intraocular pressure after vitreoretinal surgery: is early postoperative intraocular pressure elevation predictable? Retina. 2011;31(8):1545-1552.

13. Koreen L, Yoshida N, Escariao P, et al. Incidence of, risk factors for, and combined mechanism of late-onset open-angle glaucoma after vitrectomy. Retina. 2012;32(1):160-167.

14. Luk FO, Kwok AK, Lai TY, Lam DS. Presence of crystalline lens as a protective factor for the late development of open angle glaucoma after vitrectomy. Retina. 2009;29(2):218-224.

15. Yang HK, Woo SJ, Park KH, Park KH. Intraocular pressure changes after vitrectomy with and without combined phacoemulsification and intraocular lens implantation. Korean J Ophthalmol. 2010;24(6): $341-346$.
16. Mi CW, Thompson JT. Long-term follow-up of intraocular pressure after vitrectomy in eyes without preexisting glaucoma. Retina. 2015; 35(12):2543-2551.

17. Framme C, Klotz S, Wolf-Schnurrbusch UE, Wiedemann P, Wolf S. Intraocular pressure changes following $20 \mathrm{G}$ pars-plana vitrectomy. Acta Ophthalmol. 2012;90(8):744-749.

18. Jeng KW, Fine HF, Wheatley HM, Roth D, Connors DB, Prenner JL. Incidence of steroid-induced ocular hypertension after vitreoretinal surgery with difluprednate versus prednisolone acetate. Retina. 2014; 34(10):1990-1996.

19. Desai UR, Alhalel AA, Schiffman RM, Campen TJ, Sundar G, Muhich A. Intraocular pressure elevation after simple pars plana vitrectomy. Ophthalmology. 1997;104(5):781-786.

20. Al-Jazzaf AM, Netland PA, Charles S. Incidence and management of elevated intraocular pressure after silicone oil injection. J Glaucoma. 2005;14(1):40-46.

21. Nguyen QH, Lloyd MA, Heuer DK, et al. Incidence and management of glaucoma after intravitreal silicone oil injection for complicated retinal detachments. Ophthalmology. 1992;99(10):1520-1526.

22. Phelps CD, Burton TC. Glaucoma and retinal detachment. Arch Ophthalmol. 1977;95(3):418-422.

23. Gedde SJ. Management of glaucoma after retinal detachment surgery. Curr Opin Ophthalmol. 2002;13(2):103-109.

24. Honavar SG, Goyal M, Majji AB, Sen PK, Naduvilath T, Dandona L. Glaucoma after pars plana vitrectomy and silicone oil injection for complicated retinal detachments. Ophthalmology. 1999;106(1):169-176; discussion 177.

25. Van Aken E, Lemij H, Vander Haeghen Y, de Waard P. Baerveldt glaucoma implants in the management of refractory glaucoma after vitreous surgery. Acta Ophthalmol. 2010;88(1):75-79.

26. Mangouritsas G, Mourtzoukos S, Portaliou DM, Georgopoulos VI, Dimopoulou A, Feretis E. Glaucoma associated with the management of rhegmatogenous retinal detachment. Clin Ophthalmol. 2013;7: 727-734.

27. Yu AL, Brummeisl W, Schaumberger M, Kampik A, Welge-Lussen U. Vitrectomy does not increase the risk of open-angle glaucoma or ocular hypertension - a 5-year follow-up. Graefes Arch Clin Exp Ophthalmol. 2010;248(10):1407-1414.

28. Ki-I Y, Yamashita T, Uemura A, Sakamoto T. Long-term intraocular pressure changes after combined phacoemulsification, intraocular lens implantation, and vitrectomy. Jpn J Ophthalmol. 2013;57(1):57-62.

29. Lalezary M, Kim SJ, Jiramongkolchai K, Recchia FM, Agarwal A, Sternberg P Jr. Long-term trends in intraocular pressure after pars plana vitrectomy. Retina. 2011;31(4):679-685.

30. Budenz DL, Taba KE, Feuer WJ, et al. Surgical management of secondary glaucoma after pars plana vitrectomy and silicone oil injection for complex retinal detachment. Ophthalmology. 2001;108(9): $1628-1632$.
Clinical Ophthalmology

\section{Publish your work in this journal}

Clinical Ophthalmology is an international, peer-reviewed journal covering all subspecialties within ophthalmology. Key topics include: Optometry; Visual science; Pharmacology and drug therapy in eye diseases; Basic Sciences; Primary and Secondary eye care; Patient Safety and Quality of Care Improvements. This journal is indexed on Submit your manuscript here: http://www.dovepress.com/clinical-ophthalmology-journal

\section{Dovepress}

PubMed Central and CAS, and is the official journal of The Society of Clinical Ophthalmology (SCO). The manuscript management system is completely online and includes a very quick and fair peer-review system, which is all easy to use. Visit http://www.dovepress.com/ testimonials.php to read real quotes from published authors. 